\title{
MiR-125b Regulates the Osteogenic Differentiation of Human Mesenchymal Stem Cells by Targeting BMPR1b
}

\author{
Huaqing Wang ${ }^{\text {a }}$ Zhao Xie ${ }^{\mathrm{a}}$ Tianyong Hou $\mathrm{H}^{\mathrm{a}}$ Zhiqiang $\mathrm{Li}^{\mathrm{a}}$ Ke Huang ${ }^{\mathrm{a}}$

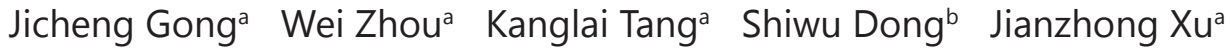

aNational \& Regional United Engineering Lab of Tissue Engineering, Department of Orthopedics, Southwest Hospital, Third Military Medical University, ${ }^{b}$ Department of Biomedical Materials Science, College of Biomedical Engineering, Third Military Medical University, Chongqing, China

\section{Key Words}

miR-125b - Human mesenchymal stem cells - Osteogenic differentiation - BMPR1b • Segmental bone defects

\begin{abstract}
Background/Aims: Osteogenic differentiation of mesenchymal stem cells (MSCs) plays a crucial role in bone regeneration and bone reparation. This complex process is regulated precisely and firmly by specific factors. Recent studies have demonstrated that miR-125b regulates osteogenic differentiation, but little is known about the molecular mechanisms of this regulation. Furthermore, how miR-125b regulates the osteogenic differentiation of MSCs still needs elucidation. Methods: In the present study, human bone marrow-derived mesenchymal stem cells (hBMSCs) were isolated and induced to osteoblasts with miR-125b inhibition or overexpression. qRT-PCR and western blot analysis were used to detect the expression of osteogenic marker genes and proteins. Alkaline phosphatase (ALP) and Alizarin Red (ARS) staining were performed to evaluate the osteoblast phenotype. TargetScan, PicTar and miRanda database were used to predict the target gene of miR-125b. Dual luciferase reporter assay and RNA interference were performed to verify the target gene. Micro-CT imaging and histochemical staining were used to investigate the bone defect repair capacity of miR-125b in vivo. Results: We observed that miR-125b was expressed at a low level during the osteogenic differentiation of hBMSCs. Then, we found that osteogenic marker genes were negatively regulated by miR-125b during the course of osteogenic differentiation, suggesting that miR$125 \mathrm{~b}$ down regulation plays an important role in the process of osteogenic differentiation. Bioinformatics approaches using miRNA target prediction algorithms indicated that the bone morphogenetic protein type Ib receptor (BMPR1b) is a potential target of miR-125b. The results of the dual luciferase reporter assay indicated that miR-125b binds to the $3^{\prime}$ UTR of the BMPR1b gene. We observed that knockdown of BMPR1b by siRNA inhibited the osteogenic differentiation of hBMSCs. Furthermore, by co-transfecting cells with an miR-125b inhibitor and si-BMPR1b, we found that the osteogenic capacity of the cells transfected with miR-125b inhibitor was blocked upon knockdown of BMPR1b. In vivo, demineralized bone
\end{abstract}

$H$. Wang and Z. Xie contributed to the work equally.

Jianzhong Xu

KARGER
Department of Orthopedics, Southwest Hospital, Third Military Medical University, Gaotanyan Street No.30, Chongqing 400038 (China)

E-Mail xjzslw@163.com 
Wang et al.: MiR-125b Suppresses Osteogenic Differentiation of hBMSCs

matrix (DBM) was composited with hBMSCs as a scaffold to repair segmental femoral defects. By inhibiting the expression of miR-125b, hBMSCs showed a better capacity to repair bone defects. Conclusions: Taken together, our study demonstrated that miR-125b regulated the osteogenic differentiation of hBMSCs by targeting BMPR1b and that inhibiting miR-125b expression could enhance the capacity of bone defect repair in vivo.

(C) 2017 The Author(s)

Published by S. Karger AG, Basel

\section{Introduction}

Human bone marrow-derived mesenchymal stem cells (hBMSCs) are a type of pluripotent stem cells derived from bone marrow; these cells were the first members of the mesenchymal stem cell (MSC) family to be discovered [1]. Upon stimulation by specific environmental factors, MSCs can be induced and differentiated into a variety of adult tissues, such as bone, fat, cartilage, nerve and muscle [2-6]. Because of their strong regenerative properties and multi-potentiality, MSCs might be used in cell therapies and tissue engineering [7-11]. As the precursor cells of osteoblasts, hBMSCs play a crucial role in either bone formation or bone differentiation and have become an optimal source of seed cells in bone tissue engineering $[12,13]$.

MicroRNAs (miRNAs) are a type of endogenous non-coding single-stranded small RNA molecule approximately 19-25 nt in length that regulates the target genes by incomplete complementarity to their nucleotides with either the 3' untranslated region (3'-UTR) or open reading frame (ORF), eventually resulting in the suppression of gene expression by inhibiting translation or promoting mRNA decay [14]. miRNAs have emerged as key regulators of diverse physiological and pathological processes, such as cell proliferation, differentiation, apoptosis and cancer $[15,16]$. As the key regulator, miRNAs also take part in the osteogenic differentiation and genesis of bone regeneration-related diseases [17, 18]. As important regulatory factors, miRNAs play a critical role in the proliferation and differentiation of stem cells. In recent years, more researchers have found that complex molecular mechanisms and intricate regulatory networks exist between MSCs and miRNAs. For instance, one study found that miR-33a-5p expression was significantly increased after TNF-alpha treatment during bone morphogenetic protein (BMP)-2-induced osteogenic differentiation of hBMSCs. Furthermore, miR-33a-5p modulates TNF-alpha-inhibited osteogenic differentiation by targeting SATB2 expression in hBMSCs [19]. A recently study demonstrated that miR-214 represses the osteogenic differentiation of MSCs by inhibiting the FGFR1/FGF signaling pathway [20]. Moreover, suppression of miR-222-3p activity promoted the osteogenic differentiation of hBMSCs through regulating the Smad5-RUNX2 signaling axis [21]. With the development of related studies, miRNAs could probably be applied in bone tissue engineering and disease therapy.

In this study, we demonstrated the negative regulatory function of miR-125b in the osteogenic differentiation of hBMSCs and investigated the expression levels of osteogenic marker genes in vitro. Furthermore, BMPR1b, an important regulator of the osteogenic differentiation of hBMSCs, was identified as a direct target of miR-125b. The results showed that down-regulating miR-125b would promote osteogenic differentiation; however, the effect of miR-125b inhibitor was blocked by knockdown of BMPR1b. Our finding suggested that miR-125b regulated the osteogenic differentiation of hBMSCs by targeting BMPR1b. Additionally; this study showed that implanting DBM-hBMSC complexes with miR-125b inhibitory function in vivo improved the bone defect repair capacity.

\section{Materials and Methods}

Isolation and culture of hBMSCs

hBMSCs used in the experiments were derived from a young healthy male volunteer. A total of 10 $\mathrm{mL}$ of bone marrow aspirate was collected from the posterior iliac crest of the volunteer under aseptic 


\section{Cellular Physiology Cell Physiol Biochem 2017;41:530-542 and Biochemistry Published \begin{tabular}{l|l} 
DOI: 10.1159/000457013 31,2017 & $\begin{array}{l}\text { C) } 2017 \text { The Author(s). Published by S. Karger AG, Basel } \\
\text { www.karger.com/cpb }\end{array}$ \\
\hline
\end{tabular}}

Wang et al.: MiR-125b Suppresses Osteogenic Differentiation of hBMSCs

conditions. Mononuclear cells were isolated from the bone marrow using Percoll solution (Sigma, USA; density, $1.073 \mathrm{~g} / \mathrm{L}$ ) and then washed twice with PBS. Cells were resuspended and cultured in Dulbecco's minimum essential medium/nutrient mixture F-12 (DMEM/F12 HyClone, USA) supplemented with 10\% fetal bovine serum (FBS, HyClone, Australia, thermoscientific.com), 2 mM L-glutamine (Sigma USA), and $100 \mathrm{U}$ penicillin/streptomycin (Sigma USA). The cells were maintained at $37^{\circ} \mathrm{C}$ in a humidified incubator with $5 \% \mathrm{CO}_{2}$. hBMSCs were separated by adherent culture and were used between the fourth and eighth passages in the experiments.

\section{Osteogenic differentiation of hBMSCs in vitro}

hBMSCs were plated at a density of $3 \times 10^{3} / \mathrm{cm}^{2}$ cells in plates. At $80 \%$ confluence, the medium was replaced with osteogenic differentiation medium containing Human Mesenchymal Stem Cell Osteogenic Differentiation Basal Medium, 10\% FBS, 1\% penicillin/streptomycin, 1\% glutamine, 0.2\% ascorbate, 1\% $\beta$-glycerophosphate, and $0.01 \%$ dexamethasone (all components purchased from Cyagen, China) to induce differentiation. The induction medium was changed every 3 days, and the hBMSCs were cultured in the induction medium for 15 days.

\section{miRNA target prediction}

Prediction of miRNA target genes was performed using the TargetScan (http://www.targetscan.org/), PicTar (http://www.pictar.org) and miRanda (http://www.microrna.org/) databases.

\section{Dual luciferase reporter assay}

A BMPR1b 3'-UTR reporter vector was synthetized by Yingrun Biotechnology (China). Either wild-type BMPR1b or its mutant fragment were inserted in the vectors (Table 1) and were termed BMPR1b-Wt and BMPR1b-Mut, respectively. The sequences of the miR-125b binding site and the mutant site are underlined.

To estimate whether miR-125b could bind to the BMPR1b 3'-UTR, 293T cells were seeded in 96-well plates. The cells were transfected with the BMPR1b-Wt or BMPR1b-Mut reporter plasmid, miRNA NC (50 $\mathrm{nM}$ ) or miR-125b mimics (50 nM) or an miR-125b inhibitor (50 nM) using Lipofectamine 2000 (Invitrogen, USA). According to the Promega protocol, cells were harvested after 48 hours, and then firefly and Renilla luciferase activities were assayed using the dual luciferase reporter assay system (Promega, USA). Renilla luciferase activity was normalized to firefly luciferase activity.

\section{Vector construction and transfection of hBMSCs}

Lentiviral vectors were constructed by Genechem (Shanghai, China). After hBMSCs were trypsinized and resuspended, they $\left(3 \times 10^{4}\right.$ cells/well) were added to 6 -well plates. Vector and polybrene were added after 24 hours. The lentiviral multiplicity of infection (MOI) was 10. An inverted fluorescent microscope was used to assess transfection efficiency after the infected hBMSCs were cultured for 72 hours.

\section{Quantitative real-time polymerase chain reaction}

Total RNA was extracted from each cell sample using TRIzol (Beyotime, China), and cDNA was synthesized using a PrimeScript RT reagent kit (TaKaRa, China) according to the manufacturer's protocol. Quantitative real-time polymerase chain reaction was performed using Premix Ex Taq (TaKaRa, China). Reactions were cycled and quantified using a 7500 Fast Real-Time PCR System (Applied Biosystems). Each sample was tested in triplicate. Relative gene expression levels of mRNA or microRNA were evaluated, and GADPH was used as an endogenous normalization control. The primer sequences of the evaluated genes are listed in Table 2.

Table 1. Sequences used in the dual luciferase reporter assay

\begin{tabular}{ll}
\hline Name & Forward $\left(5^{\prime}\right.$-3' $\left.^{\prime}\right)$ \\
\hline BMPR1b & TCTTCTGTTTGTGGGCAGAGCAAAAGACATCAAATAAGCATCCACAGTACAAGCCTTGAACATCGTCC \\
3'-UTR-WT & TGCTTCCCAGTGGGTTCAGACCTCACCTCTCAGGGAGCGACCTGGGCAAAGACAGAGAAGCTCCCAGA \\
& AGGAGAGATTGATCCATGTCTGTTTGTAGGACGGAGAAACCGCTTGGGTAA \\
BMPR1b & TCTTCTGTTTGTGGGCAGAGCAAAAGACATCAAATAAGCATCCACAGTACAAGCCTTGAACATCGTCC \\
3'-UTR-Mut & TGCTTCCCAGTGGGTTCAGACCTCACCTCTGTCGGAGCGACCTGGGCAAAGACAGAGAAGCTCCCAGA \\
& AGGAGAGATTGATCCATGTCTGTTTGTAGGACGGAGAAACCGCTTGGGTAA \\
\hline
\end{tabular}




\section{Cellular Physiology Cell Physiol Biochem 2017;41:530-542

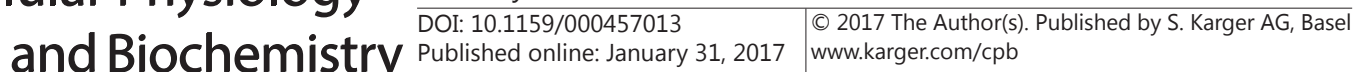

Wang et al.: MiR-125b Suppresses Osteogenic Differentiation of hBMSCs

Table 2. Primers used in qRT-PCR

\begin{tabular}{lll}
\hline Gene & Sense primer $\left(5^{\prime}-3^{\prime}\right)$ & antisense primer $\left(3^{\prime}-5^{\prime}\right)$ \\
\hline Runx2 & ACTTCCTGTGCTCGGTGCT & GACGGTTATGGTCAAGGTGAA \\
OSX & TCTCCCTCCCTCTCCCTTT & ATCATTAGCATAGCCTGAG \\
OCN & CCTCACACTCCTCGCCCTAT & GTGGTCAGCCAACTCGTCAC \\
GAPDH & TCCATGACAACTTTGGTATCG & TGTAGCCAAATTCGTTGTCA \\
BMPR1b & AAGGCTCAGATTTTCAGTGTCG & GAGGCAGTGTAGGGTGTAGGTC \\
miR-125b & TCCCTGAGACCCTAACTTGTGA & GCGAGCACAGAATTAATACGAC \\
\hline
\end{tabular}

Western blot analysis

After cells were washed twice with PBS, MSC lysates for Western blotting were extracted with RIPA lysis buffer (Beyotime, China) supplemented with phenylmethylsulfonyl fluoride (PMSF), and the protein concentration of the lysate was determined using a BCA protein assay kit (Beyotime, China). Equal amounts of protein samples were separated on a gel by SDS-PAGE and transferred to polyvinylidene fluoride (PVDF) membranes (Millipore Corporation, Billerica, MA). After the membranes were blocked with 5\% non-fat milk for 1 hours, the membranes were incubated with primary antibodies against Runx2, OSX, OCN, and BMPR1b (Abcam, UK) as well as $\beta$-actin (Beyotime, China) according to the manufacturer's instructions. Then, the membranes were incubated with a horseradish peroxidase-conjugated goat anti-rabbit IgG secondary antibody (Beyotime, China) for 2 hours. Bands were detected using ECL (Beyotime, China). Protein levels were quantified by densitometry using Quantity One software (Bio-Rad Laboratories, Munich, Germany).

\section{Alkaline phosphatase staining, Alizarin Red staining and ALP assay}

Cells were washed twice with PBS and fixed in 4\% polyoxymethylene for 10 minutes. ALP staining was performed using a BCIP/NBT Alkaline Phosphatase Color Development kit (Beyotime, China) according to the instructions of the manufacturer. After the cells were fixed and washed, Alizarin red S staining solution (Cyagen, China) was added for 5 minutes. The samples were rinsed well 3 times with PBS and then imaged. hBMSCs were collected into a 96-well plate, and quantitative ALP measurements were performed using an Alkaline Phosphatase Assay kit (Nanjing Jiancheng Bioengineering Institute, China). ALP activity was determined at a wavelength of $520 \mathrm{~nm}$.

\section{Animals and surgical procedures}

Ten 7-week-old male BALB/c nude mice weighing 16-23.5 g were used. All animal procedures were approved by the Institutional Animal Care and Use Committee of the Third Military Medical University. Before surgery, cut the DBMs (Datsing Biological Technology Co., Ltd, China) into $2 \mathrm{~mm} \times 1.5 \mathrm{~mm} \times 1.5 \mathrm{~mm}$ and seed cells $\left(3 \times 10^{4} / \mathrm{ml}\right)$ one day in advance. The animals were anesthetized with an intraperitoneal injection of sodium pentobarbital (Sigma, USA, $45 \mathrm{mg} / \mathrm{kg}$ ). The skin of the surgical areas was disinfected with $0.5 \%$ iodine and prepared for aseptic surgery. An 8-mm incision was made on the anterolateral thigh, and the vastus lateralis and biceps femoris were separated to expose the femur. The periosteum was incised along the length of the femur and then stripped. A $2 \mathrm{~mm} \times 1.5 \mathrm{~mm}$ segmental bone defect was created by applying a dental drill in the middle segment of femur, and bone grafts were used to fill in the defects. The wound was closed in layers with 6-0 sutures.

\section{Micro-CT imaging}

After the animals were euthanized, the areas of the femurs were imaged using a micro-computed tomography imager (SkyScan 1272, Bruker micro-CT, Kontich, Belgium). The images were scanned with a voxel size of $14 \mu \mathrm{m}$, a tube voltage of $70 \mathrm{KV}$, and a tube current of $114 \mu \mathrm{A}$. Images were reconstructed using micro-CT bundled software (GPU-based NRecon V1.6.9.8, Bruker micro-CT, Kontich, Belgium) and then analyzed using CTAn software (V1.14.10.4, Bruker micro-CT, Kontich, Belgium). Parameters including bone mineral density (BMD) and trabecular number (Tb.N) were calculated.

\section{Paraffin section processing and histochemical staining}

The animals were euthanatized at eight weeks after surgery. Femurs and the neighboring tissue were collected. The samples were fixed in $4 \%$ paraformaldehyde, decalcified with 10\% EDTA and gradually dehydrated with ethanol. For histochemical analysis, samples were embedded in paraffin and sectioned. Finally, HE and Masson trichrome staining were performed to detect cells and bone structure. 
Statistical analysis

Data are presented as the mean \pm SD. Statistical significance was determined using a paired-samples t-test using SPSS 19.0, and $\mathrm{p}<0.05$ was considered statistically significant.

\section{Results}

miR-125b inhibits osteogenic differentiation of $h B M S C s$

During the osteogenic differentiation of hBMSCs, the expression level of miR-125b was determined by qRT-PCR at different time points. As shown in Fig. 1A, the expression of miR$125 \mathrm{~b}$ decreased by more than $40 \%$ on the $3^{\text {rd }}$ day of induction compared with that of miR$125 \mathrm{~b}$ at day 0 and remained down-regulated until day 14 . Subsequently, the expression level rebounded at day 21 but was still at a lower level compared with the pre-induction level. This finding suggests that miR-125b might down-regulate osteogenic differentiation.

To evaluate the effects of different lentiviral vectors on miR-125b expression in hBMSCs, an miR-125b lentiviral vector, an miR-125b inhibitor and their respective negative controls were transfected into hBMSCs. hBMSCs were harvested at different time points, and miR$125 \mathrm{~b}$ expression levels were detected by qRT-PCR. Mature miR-125b levels were elevated

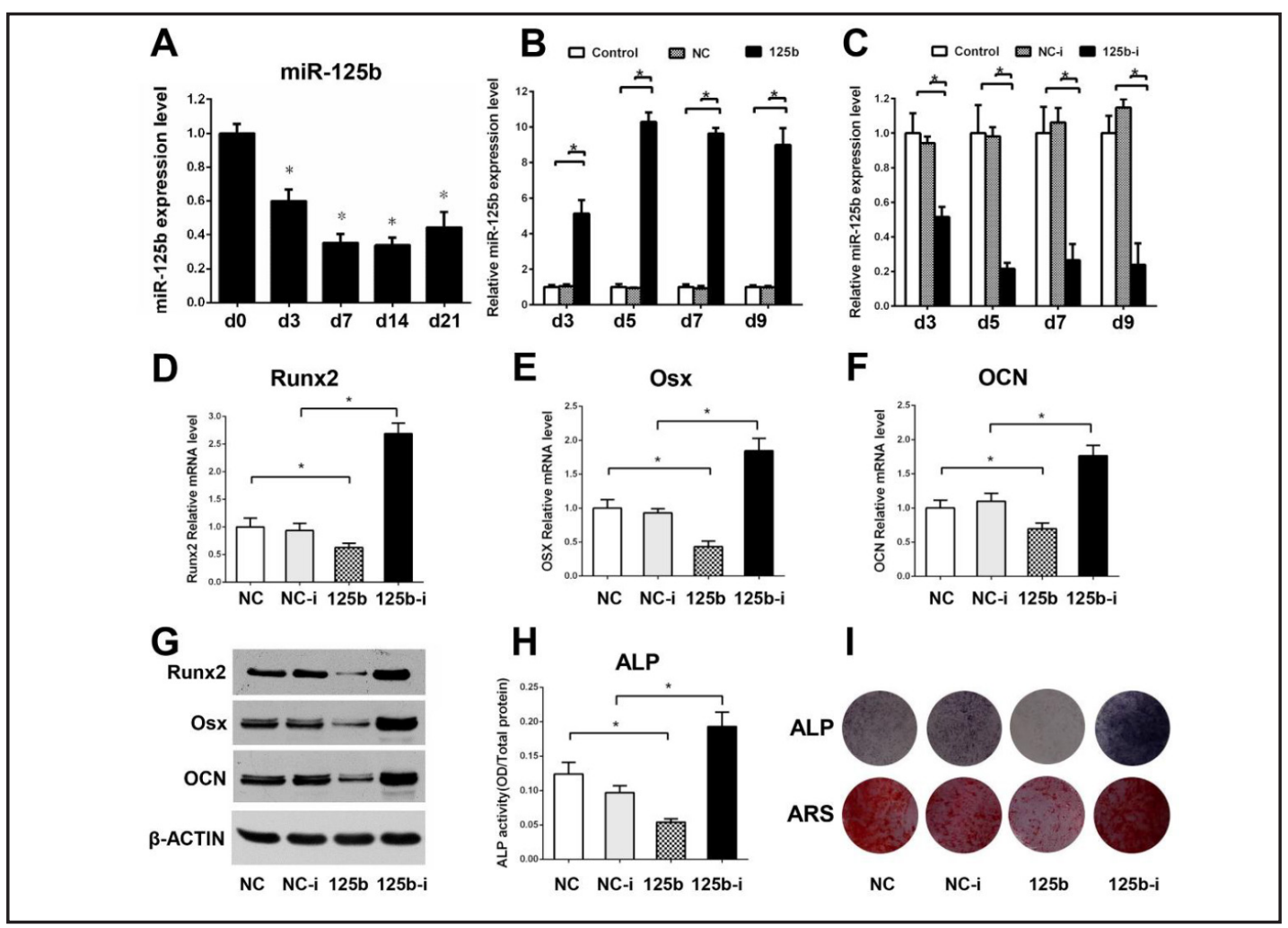

Fig. 1. miR-125b inhibited the osteogenic differentiation of hBMSCs. (A) miR-125b expression levels were detected by qRT-PCR during the osteogenic differentiation of hBMSCs at different time points; (B) qRT-PCR detection of the miR-125b expression levels after hBMSCs were transfected with miR-125b lentiviral vector or a NC empty vector; (C) miR-125b expression levels after hBMSCs were transfected with an miR-125b inhibitor and or a NC empty vector; qRT-PCR detection of the mRNA expression levels of osteoblast-specific genes, including Runx2 (D), Osx (E) and OCN (F), on the $5^{\text {th }}$ day after transfection and induction of osteogenic differentiation; $(G)$ Western blot analysis for marker genes was performed to investigate the effect of miR-125b on Runx2, Osx and OCN protein expression during the osteogenic differentiation of hBMSCs. (H) ALP activity was also measured under the same conditions; (I) ALP staining was performed on day 7, and ARS staining was performed on day 21 to show that ALP activity in and calcification of cells undergoing osteogenic differentiation were negatively regulated by miR-125b. $(* p<0.05)$.

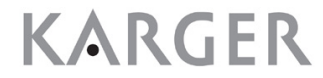


four-fold by miR-125b lentiviral vector relative to control at day 3 post-transfection, with the levels maintained at 10-, 9- and 8-fold higher at 5, 7 and 9 days, respectively (Fig. 1B). Conversely, 1.9-, 4.7-, 3.7- and 4.2-fold reductions of endogenous miR-125b expression were shown after 3, 5, 7 and 9 days, respectively, following miR-125b inhibitor transfection (Fig. 1C). The corresponding negative controls had a minimal effect on miR-125b content.

To study the impact of miR-125b on the osteogenic differentiation of hBMSCs, cells were induced toward osteogenic differentiation after being transfected with an miR-125b lentiviral vector or an miR-125b inhibitory lentiviral vector as well as their respective negative controls. As determined by qRT-PCR, the mRNA expression levels of some osteoblast-specific genes, including Runx2 (Fig. 1D), Osx (Fig. 1E) and OCN (Fig. 1F) were significantly downregulated on the $5^{\text {th }}$ day after transfection but upregulated after inhibition of miR-125b. Meanwhile, the corresponding expression of proteins encoded by these genes was verified by Western blot (Fig. 1G), and the Western results were consistent with the qRT-PCR results. Similar results were also observed for ALP activity (Fig. 1H). The osteoblast phenotype was evaluated by determining ALP staining and ARS staining. Overexpression of miR-125b significantly reduced osteogenic differentiation, as indicated by decreased ALP activity (Fig. 1I, upper) and reduced in vitro calcium deposition as visualized by ARS staining (Fig. 1I, lower) in miR-125b-transfected hBMSCs compared with cells transfected with empty vector as a negative control. In contrast, ALP activity and calcium deposition were enhanced in miR125b inhibitor-treated hBMSCs.

The 3'-UTR of BMPR1b is a direct target of miR-125b

To investigate the molecular mechanisms that underlie xmiR-125b-mediated regulation of osteogenic differentiation, we searched for potential targets of miR-125 related to osteogenic differentiation using a bioinformatics approach with miRNA target prediction algorithms and TargetScan, PicTar and miRanda databases. We identified bone morphogenetic protein type $1 \mathrm{~b}$ receptor (BMPR1b), which is a kinase receptor of BMPs that has been confirmed to play an important role in the course of osteogenic differentiation, as a potential target among the predicted candidates.

To investigate whether miR-125b could impact the expression of BMPR1b, hBMSCs were divided into five groups: miR-125b, 125b-NC, 125b-inhibitor, NC-inhibitor and control. According to the qRT-PCR (Fig. 2A) and Western blot analyses (Fig. 2B), the expression levels of the mRNA and protein of BMPR1b were significantly reduced in the miR-125b group compared with the 125b-NC or control groups on day 5 after osteogenic differentiation was induced. Conversely, down-regulation of miR-125b could increase the BMPR1b mRNA and protein contents. The expression of BMPR1b in either the 125b-NC or NC-inhibitor group minimally differed compared to the control group.

According to the predictions of TargetScan, the targeting site in the 3'-UTR of BMPR1b is partially complementary to miR-125b (Fig. 2C). We hypothesize that miR-125b binds to the 3'-UTR of the BMPR1b gene, thus inhibiting the gene expression of BMPR1b. 293T cells were divided into three groups (Table 3), and the dual luciferase reporter assay was performed to verify this hypothesis. As shown in Fig. 2D, the relative luciferase activity was decreased in the mimics group compared with the NC group but enhanced in the inhibitor group. To further confirm that miR-125b inhibits BMPR1b by binding to the 3'-UTR of the gene, a BMPR1b mutant reporter plasmid was constructed, and the cells were divided into three groups (Table 4). The dual luciferase activity assay detected that the relative luciferase activity of the BMPR1b-Wt group decreased compared with the NC group, but the BMPR1bMut group seemed to have hardly any difference compared to the NC group regarding luciferase activity (Fig. 2E). The results indicated that BMPR1b is a direct target of miR-125b.

Knockdown of BMPR1b inhibits the osteogenic differentiation of hBMSCs

qRT-PCR and Western blot analyses were used to investigate BMPR1b expression levels at different time points during the course of osteogenic differentiation of hBMSCs. The mRNA expression levels increased by $31 \%$ on day 3 and were over 2 -fold higher on day 7 as KARGER 


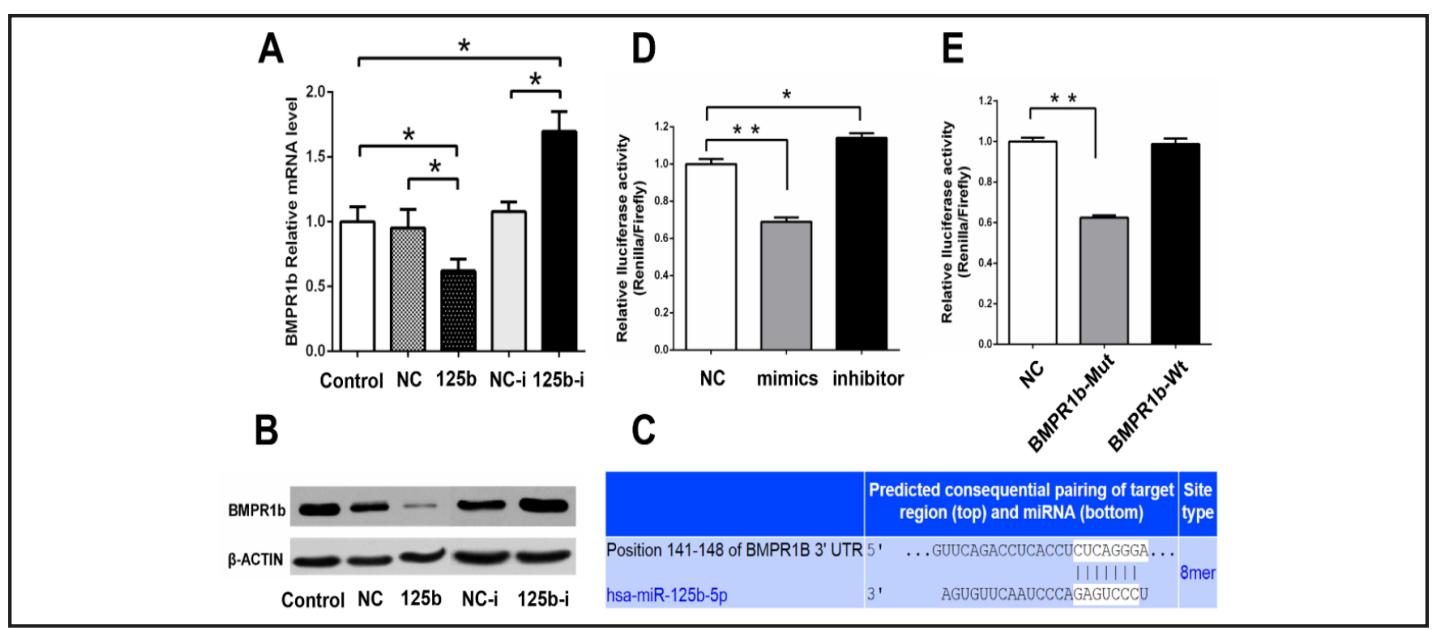

Fig. 2. The 3'-UTR of BMPR1b is a direct target of miR-125b. (A) BMPR1b gene expression levels were detected by qRT-PCR on day 5 after transfected or untransfected cells were induced toward osteogenic differentiation. (B) Western blot analysis of BMPR1b protein expression was performed on day 5 after different cell treatment groups were induced toward osteogenic differentiation. (C) miR-125b is partially complementary to a region in the BMPR1b 3'-UTR. (D) Wild-type BMPR1b 3'-UTR reporters were co-transfected with either miR-125b-mimics or miR-125b-inhibitor into 293T cells, and the dual luciferase reporter assay was performed. (E) Wild-type BMPR1b 3'-UTR reporters or mutated BMPR1b 3'-UTR reporters were co-transfected with miR-125b-mimics into 293T cells, and the dual luciferase reporter assay was performed. All of these data were averaged from three independent experiments, and the firefly luciferase activity was normalized to the Renilla luciferase activity as a control. ${ }^{*} p<0.05,{ }^{* *} p<0.01$.

Table 3. Dual luciferase reporter assay groups

\begin{tabular}{ll}
\hline Group & Contents of co-transfected 293T cells \\
\hline NC & BMPR1b reporter + miR-NC \\
mimics & BMPR1b reporter + miR-125b-mimics \\
inhibitor & BMPR1b reporter + miR-125b-inhibitor \\
\hline
\end{tabular}

Table 4. Dual luciferase reporter assay groups (mutated reporter)

\begin{tabular}{ll}
\hline Group & Contents of co-transfected 293T cells \\
\hline NC & empty reporter + miR-125b-mimics \\
BMPR1b-Wt & BMPR1b-Wt reporter + miR-125b-mimics \\
BMPR1b-Mut & BMPR1b-Mut reporter + miR-125b-mimics \\
\hline
\end{tabular}

determined by qRT-PCR analysis (Fig. 3A). Western blotting also showed similar results (Fig. 3B). The results suggested that BMPR1b might be involved in the osteogenic process. Then, RNA interference was used to study whether BMPR1b could negatively regulate osteogenic differentiation. Cells were transfected with either si-BMPR1b or si-NC and then induced toward osteogenic differentiation. ALP activity was decreased upon knockdown of BMPR1b by RNA interference (Fig. 3C). qRT-PCR analysis demonstrated that the mRNA expression levels of the osteogenic-specific genes Runx2 (Fig. 3D), Osx (Fig. 3E) and OCN (Fig. 3F) were significantly decreased in the si-BMPR1b group compared with the si-NC or control groups; these findings were also in agreement with the Western blot results for the corresponding protein (Fig. 3G). Similarly, the osteoblast phenotype revealed that BMPR1b knockdown could significantly inhibit osteogenic differentiation, which was represented as decreased ALP activity (Fig. 3H, upper) and reduced in vitro calcium deposition as measured by ARS staining (Fig. 3H, lower).

\section{BMPR1b knockdown blocks the effect of miR-125b}

As shown in Fig. 3A, the content of BMPR1b peaked on day 7 during the osteogenesis differentiation of hBMSCs, while the expression of miR-125b was concurrently at a lower 


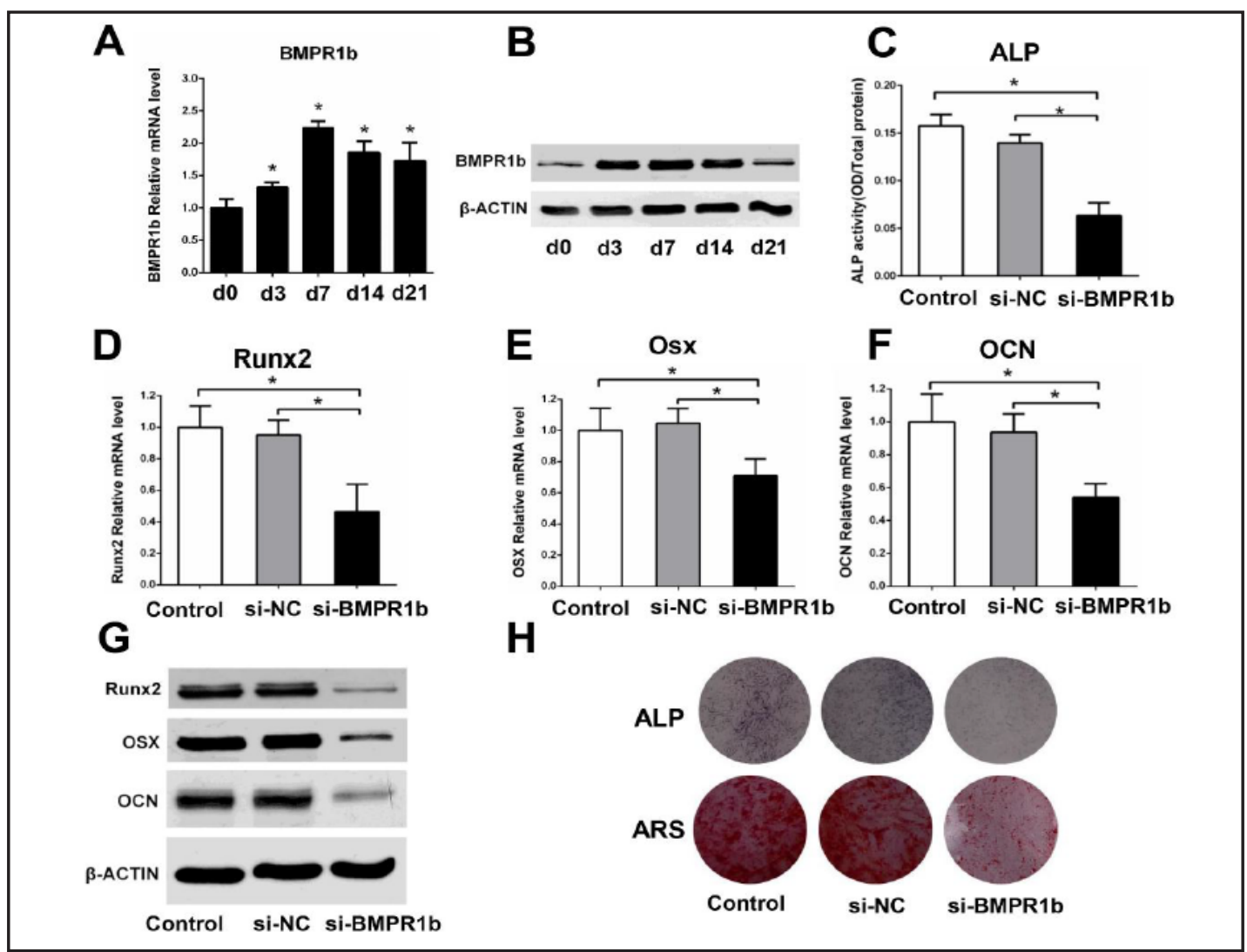

Fig. 3. Knockdown of BMPR1b inhibits the osteogenic differentiation of hBMSCs. (A) qRT-PCR was used to investigate BMPR1b gene expression on days $0,3,7,14$ and 21 of the osteogenic differentiation of hBMSCs. (B) BMPR1b protein expression in hBMSCs during the course of osteogenic differentiation as determined by Western blot analysis. (C) ALP activity was decreased in the si-BMPR1b group on day 7 of osteogenic differentiation. (D-F) qRT-PCR detection of the mRNA expression levels of the osteogenic marker genes Runx2 (D), Osx (E) and OCN (F) on day 7 of osteogenic differentiation. (G) Western blot analysis was performed to investigate the effect of BMPR1b on the expression of proteins encoded by osteogenic marker genes. $(\mathrm{H})$ ALP staining was performed on day 7, and ARS staining was performed on day 21 to show that ALP activity and calcification, respectively, were inhibited upon BMPR1b knockdown. $\left({ }^{*} p<0.05\right)$.

level (Fig. 1A). Combined with the aforementioned results, we could speculate that miR-125b should have some relationship with BMPR1b during osteogenic differentiation. To clarify the relationship between miR-125b and BMPR1b, hBMSCs were co-transfected with an miR$125 \mathrm{~b}$ inhibitor, which has previously been shown to promote osteogenesis, and si-BMPR1b, which could inhibit osteogenesis. Then, co-transfected cells were induced toward osteogenic differentiation. As shown by qRT-PCR analysis of osteogenic marker mRNA expression (Fig. 4 A-C), Western blot analysis of osteogenic marker protein expression (Fig. 4D), ALP activity assay (Fig. 4E), and ALP and ARS staining (Fig. 4F), cells co-transfected with either miR$125 \mathrm{~b}$ inhibitor or si-NC could still promote osteogenesis, but after knockdown of BMPR1b, inhibition of miR-125b could no longer improve osteoblast differentiation. These results indicate that knockdown of BMPR1b would influence the osteogenic capacity of the miR$125 \mathrm{~b}$ inhibitor. In conclusion, miR-125b regulated the osteogenic differentiation of hBMSCs by targeting BMPR1b.

Down-regulating miR-125b promotes the repair of segmental bone defects in vivo

To determine whether an miR-125b inhibitor could enhance the osteogenic capacity of hBMSCs in vivo, an established animal model of bilateral segmental femoral defects with athymic nude mice was used. Demineralized bone matrixes (DBMs) were selected as 


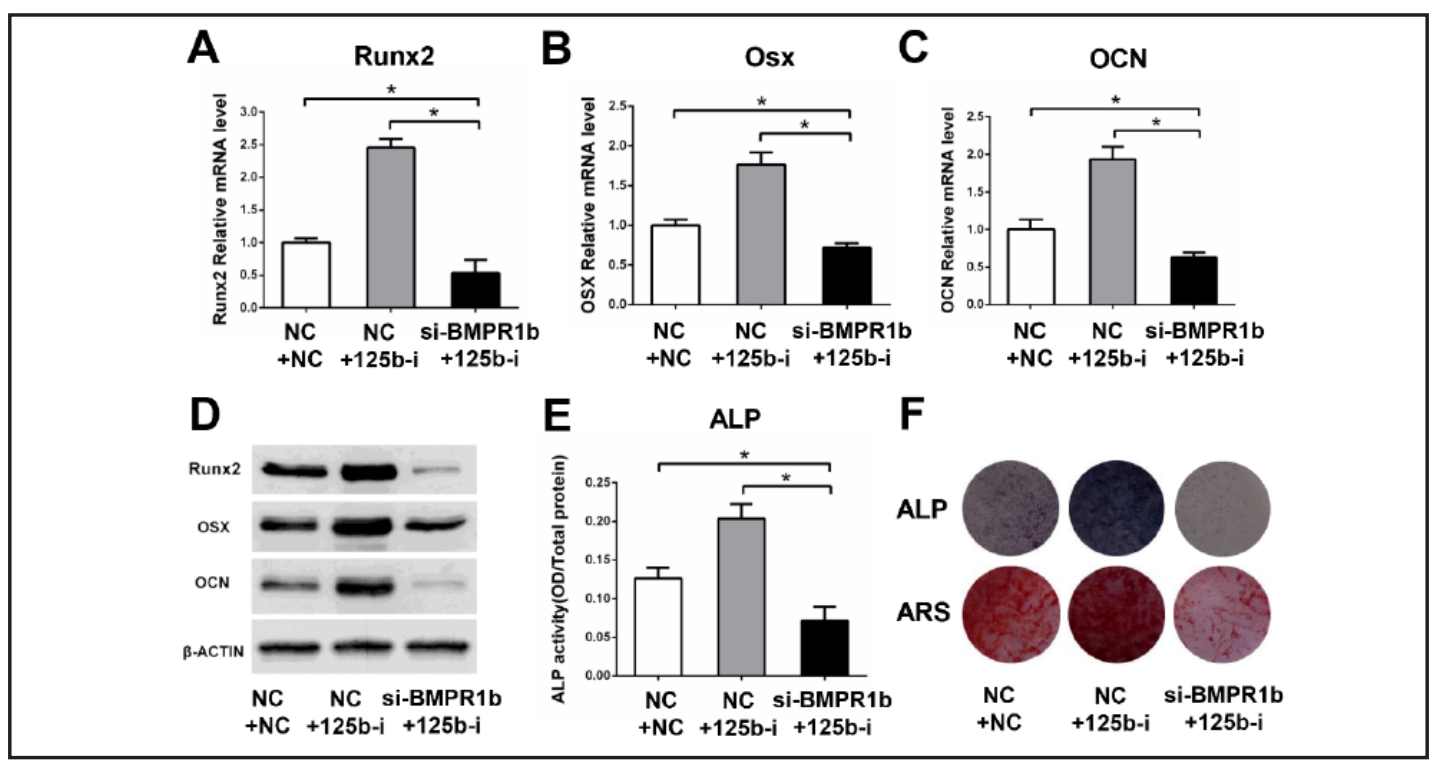

Fig. 4. BMPR1b knockdown blocks the effect of miR-125b. (A-C) qRT-PCR detected the mRNA expression levels of the osteogenic marker genes Runx2 (A), Osx (B) and OCN (C) on day 7 of osteogenic differentiation. (D) Western blot analysis was performed to investigate the effect of BMPR1b knockdown on the expression of proteins encoded by these osteogenic marker genes. (E) ALP activity was decreased in the group co-transfected with si-BMPR1b and miR-125b-inhibitor on day 7 of osteogenic differentiation. (H) ALP staining was performed on day 7 and ARS staining was performed on day 21 to show that ALP activity and calcification, respectively, were inhibited on the condition of BMPR1b knockdown even though cells were transfected with miR-125b-inhibitor. $(* p<0.05)$.

the scaffold and transplanted to areas of bone defects. hBMSCs transfected with the miR$125 \mathrm{~b}$ inhibitor and untransfected control cells were seeded in the DBM and then surgically transplanted into the defective regions. Micro-CT scans were performed 8 weeks after implantation, and the efficiency of bone defect repair was quantified with BMD and the Tb.N. As shown in Fig. 5A, regardless of experimental side or control side, bone defects were all repaired with grafts, but the micro-CT images showed a more compact structure of trabecular bone in the bone defect region on the experimental side (Fig. 5A, right). On the experimental side, the BMD was increased by 51\% (Fig. 5B), and the Tb.N was increased by $79 \%$ (Fig. 5C) compared to those on the control side. These results suggested that the experimental side had better bone regenerative capacity than the control side.

Histochemical staining, including HE (Fig. 5D, upper) and Masson trichrome staining (Fig. 5D, lower), were performed to investigate new bone generation and bone maturity. As shown by HE staining, more trabecular bone and osteoblasts (arrows) were observed on the experimental side. By Masson trichrome staining, more mature bone structures were stained red (arrows) on the experimental side compared with the control side. These results suggest that down-regulating miR-125b promotes bone regeneration and accelerates bone maturity in the process of repairing bone defects with DBM-hBMSC complexes.

\section{Discussion}

As human mesenchymal stem cells (hMSCs) can be induced toward osteoblastic cells under appropriate conditions by specific osteoinductive factors, they have been regarded as one of the most important seeding cells in bone tissue engineering [9-11]. miRNAs have been reported to be critical regulatory factors in the osteogenic differentiation of hBMSCs [22-24]. Past research [25] has shown that miR-125b was involved in the osteogenic differentiation of ST2 cells through down-regulation of cell proliferation. Our research team has reported that 


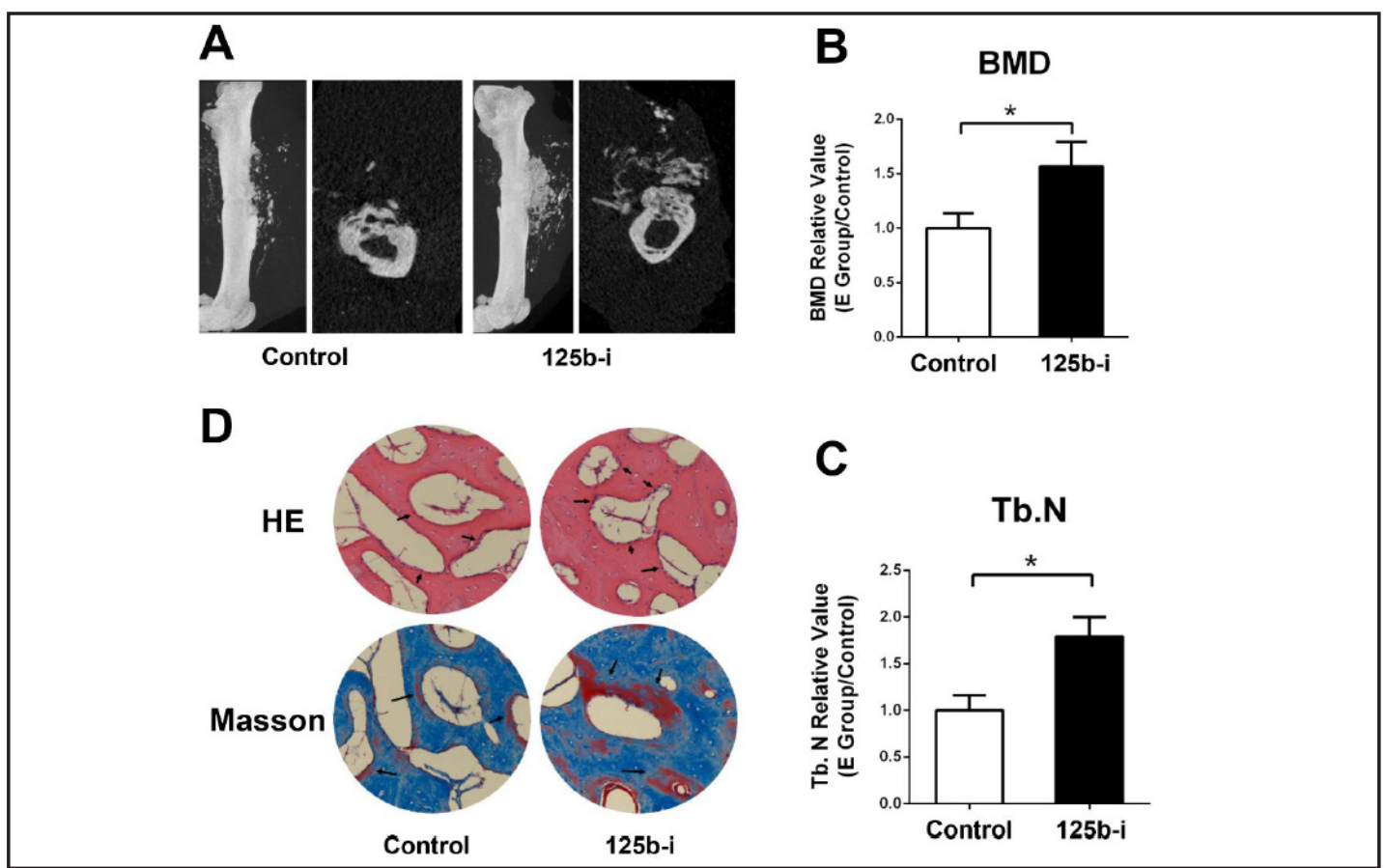

Fig. 5. Down-regulating miR-125b promotes the repair of segmental bone defects in vivo. (A) Micro-CT scan showed that regardless of the experimental side or the control side, all of the bone defects were repaired with grafts, while the experimental side showed a better repair capacity than the control side. (B-C) At 8 weeks after surgery, a micro-CT scan was performed, and the BMD (A) and Tb.N (B) of the defect areas on the experimental side were significantly higher than those of the control side. ${ }^{*} p<0.05$ (D) The repair and regeneration of grafts in two groups were evaluated by HE staining (arrow showed new bone generation) and Masson trichrome staining (arrow showed mature bone) of paraffin sections.

miR-125b is a key regulatory factor of osteogenic differentiation in C3H10T1/2 cells [26]. However, little is known about the details of the influence of miR-125b on the osteogenic differentiation of hBMSCs in vitro and vivo. In this study, we identified miR-125b as a negative regulator of hBMSC osteoblast differentiation. The in vitro experimental results revealed that inhibition of miR-125b function enhanced the osteoblast differentiation of hBMSCs, whereas overexpression of miR-125b inhibited osteogenic differentiation. Furthermore, the in vivo experiments showed that by inhibiting miR-125b with antimiR-125b, the scaffold materials promote new bone formation and the repair of the bone defect. These results suggest that miR-125b plays an important role in bone formation in vitro and in vivo by negatively regulating the osteogenic differentiation of hMSCs and further enhancing the healing of bone defects in vivo.

BMPs are members of the transforming growth factor- $\beta$ (TGF- $\beta$ ) superfamily [27, 28]. Two types of serine/threonine kinase receptors are required for signal transduction, including type I and type II receptors that bind to BMPs [29], and both type I and type II receptors are required for signal transduction[28]. Some important members of BMPs such as BMP2, BMP4, BMP6, BMP7, BMP10 and BMP14 have been confirmed in previous studies and are of great significance in skeletal repair and regeneration [30-33]. BMPR1b, a receptor of the aforementioned BMPs, has already been proven to play an important role in the osteogenic differentiation of MSCs [34,35]. A previous study had observed that the increase in miR-125b expression was attenuated in osteoblastic-differentiated ST2 cells induced by BMP-4 [25].

Most of the research about miR-125b mainly focused on the proliferation and migration of tumor cells $[36,37]$. While some researchers observed that miR-125b inhibits osteogenic 
differentiation of MSCs, little is known about its target gene and the molecular mechanisms involved in this process. Using a bioinformatics approach with miRNA targeting prediction algorithms, we found that BMPR1B might be the target gene of miR-125b. This assumption has been subsequently verified by dual luciferase reporter assay, by which we found that miR-125b binds to the 3'-UTR of the BMPR1b gene. Therefore, we hypothesized that miR$125 \mathrm{~b}$ may down-regulate BMPR1b in the process of hBMSC osteoblast differentiation. To confirm this hypothesis, we used siRNA to block the expression of BMPR1b. The results showed that the osteogenic differentiation of hBMSCs was inhibited by down-regulating the expression of BMPR1b. Furthermore, the cells were co-transfected with si-BMPR1b and miR-125b inhibitor were observed to no longer promote the osteogenic differentiation of hBMSCs because the BMPR1b expression had been blocked by siRNA.

Demineralized bone matrix (DBM) is bone that has been acid-treated to remove the mineralized portion while maintaining the organic matrix and growth factors [38] and is usually derived from human allograft tissue. As a type of allograft, DBM has osteoconductive and osteoinductive properties that prompt bone regeneration and promote bone defect repair. The demineralization process destroys the antigenic materials in bone, making it less immunogenic than mineralized allografts $[39,40]$. Currently, DBM is widely used in multiple medical applications [41-43] such as spinal fusions, skeletal defect repair and craniomaxillofacial surgeries. As DBM has no osteogenesis properties, it is commonly composited with cytokines or mesenchymal stem cells to improve the potential of enhanced bone regeneration $[44,45]$. In this study, we selected DBM as the scaffold and hBMSCs as the seed cells. The reparative capacity of hBMSCs transfected with miR-125b inhibitor on bone defects were compared with that of the untransfected cells in vivo. Our results showed that the DBM-hBMSCs complexes with miR-125b inhibition had better capacity to repair defects in vivo, which was embodied by a higher bone mineral density and more trabecular bones in the defective areas. Moreover, bone regeneration and maturity was also increased on the experimental side. Combined with the previous results, we speculated that downregulating miR-125b would increase the content of osteogenic factors in the bone defect regions such that the osteogenesis was enhanced. The results provide the possibilities for the modification and improvement of engineered bone tissue grafts.

In summary, our results demonstrate that miR-125b functions as a negative regulator of osteogenic differentiation of hBMSCs by directly binding to the 3'-UTR of the BMPR1b gene. Importantly, our results show that down-regulating miR-125b improves bone regeneration and maturity in vivo, suggesting that the role of miR-125b in the regenerative capacity of hBMSCs offers a new therapeutic potential in bone tissue engineering.

\section{Acknowledgments}

This work was supported by the Foundation of Southwest Hospital (SWH2013JS07) and the Military Foundation (BWS11C040).

\section{Disclosure Statement}

The authors declare no conflict of interest

\section{References}

1 Friedenstein AJ, Petrakova KV, Kurolesova AI, Frolova GP: Heterotopic of bone marrow. Analysis of precursor cells for osteogenic and hematopoietic tissues. Transplantation 1968;6:230-247.

2 Haynesworth SE, Goshima J, Goldberg VM, Caplan AI: Characterization of cells with osteogenic potential from human marrow. Bone 1992;13:81-88. 


\section{Cellular Physiology Cell Physiol Biochem 2017;41:530-542

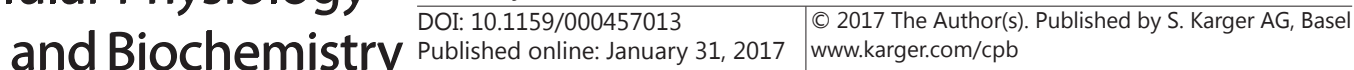

Wang et al.: MiR-125b Suppresses Osteogenic Differentiation of hBMSCs

- 3 Dennis JE, Merriam A, Awadallah A, Yoo JU, Johnstone B, Caplan AI: A quadripotential mesenchymal progenitor cell isolated from the marrow of an adult mouse. J Bone Miner Res 1999;14:700-709.

-4 Yoo JU, Barthel TS, Nishimura K, Solchaga L, Caplan AI, Goldberg VM, Johnstone B: The chondrogenic potential of human bone-marrow-derived mesenchymal progenitor cells. J Bone Joint Surg Am 1998;80:1745-1757.

5 Kopen GC, Prockop DJ, Phinney DG: Marrow stromal cells migrate throughout forebrain and cerebellum, and they differentiate into astrocytes after injection into neonatal mouse brains. Proc Natl Acad Sci USA 1999;96:10711-10716.

-6 Wakitani S, Saito T, Caplan AI: Myogenic cells derived from rat bone marrow mesenchymal stem cells exposed to 5-azacytidine. Muscle Nerve 1995;18:1417-1426.

7 Allers C, Lasala GP, Minguell JJ: Presence of osteoclast precursor cells during ex vivo expansion of bone marrow-derived mesenchymal stem cells for autologous use in cell therapy. Cytotherapy 2014;16:454-459.

8 Watson L, Elliman SJ, Coleman CM: From isolation to implantation: A concise review of mesenchymal stem cell therapy in bone fracture repair. Stem Cell Res Ther 2014;5:51.

9 Ng J, Bernhard J, Vunjak-Novakovic G: Mesenchymal stem cells for osteochondral tissue engineering. Methods Mol Biol 2016;1416:35-54.

10 TheinHan W, Liu J, Tang M, Chen W, Cheng L, Xu HH: Induced pluripotent stem cell-derived mesenchymal stem cell seeding on biofunctionalized calcium phosphate cements. Bone Res 2013;4:371-384.

11 See EY, Toh SL, Goh JC: Multilineage potential of bone-marrow-derived mesenchymal stem cell cell sheets: Implications for tissue engineering. Tissue Eng Part A 2010;16:1421-1431.

-12 Hidalgo-Bastida LA, Cartmell SH: Mesenchymal stem cells, osteoblasts and extracellular matrix proteins: Enhancing cell adhesion and differentiation for bone tissue engineering. Tissue Eng Part B Rev 2010;16:405-412.

13 Shimizu K, Ito A, Yoshida T, Yamada Y, Ueda M, Honda H: Bone tissue engineering with human mesenchymal stem cell sheets constructed using magnetite nanoparticles and magnetic force. J Biomed Mater Res B Appl Biomater 2007;82:471-480.

14 Huntzinger E, Izaurralde E: Gene silencing by micrornas: Contributions of translational repression and mrna decay. Nat Rev Genet 2011;12:99-110.

15 Qiao W, Chen L, Zhang M: Microrna-205 regulates the calcification and osteoblastic differentiation of vascular smooth muscle cells. Cell Physiol Biochem 2014;33:1945-1953.

16 Esteller M: Non-coding rnas in human disease. Nat Rev Genet 2011;12:861-874.

17 Li J, Zhang Y, Zhao Q, Wang J, He X: Microrna-10a influences osteoblast differentiation and angiogenesis by regulating beta-catenin expression. Cell Physiol Biochem 2015;37:2194-2208.

18 You L, Pan L, Chen L, Gu W, Chen J: Mir-27a is essential for the shift from osteogenic differentiation to adipogenic differentiation of mesenchymal stem cells in postmenopausal osteoporosis. Cell Physiol Biochem 2016;39:253-265.

19 Mi W, Shi Q, Chen X, Wu T, Huang H: Mir-33a-5p modulates tnf-alpha-inhibited osteogenic differentiation by targeting satb2 expression in hbmscs. FEBS Lett 2016;590:396-407.

20 Yang L, Ge D, Cao X, Ge Y, Chen H, Wang W, Zhang H: Mir-214 attenuates osteogenic differentiation of mesenchymal stem cells via targeting fgfr1. Cell Physiol Biochem 2016;38:809-820.

-21 Yan J, Guo D, Yang S, Sun H, Wu B, Zhou D: Inhibition of mir-222-3p activity promoted osteogenic differentiation of hbmscs by regulating smad5-runx2 signal axis. Biochem Biophys Res Commun 2016;470:498-503.

22 Zhang JF, Fu WM, He ML, Xie WD, Lv Q, Wan G, Li G, Wang H, Lu G, Hu X, Jiang S, Li JN, Lin MC, Zhang YO, Kung HF: Mirna-20a promotes osteogenic differentiation of human mesenchymal stem cells by coregulating bmp signaling. RNA Biol 2011;8:829-838.

23 Baglio SR, Devescovi V, Granchi D, Baldini N: Microrna expression profiling of human bone marrow mesenchymal stem cells during osteogenic differentiation reveals osterix regulation by mir-31. Gene 2013;527:321-331.

24 Huang J, Meng Y, Liu Y, Chen Y, Yang H, Chen D, Shi J, Guo Y: Microrna-320a regulates the osteogenic differentiation of human bone marrow-derived mesenchymal stem cells by targeting hoxa10. Cell Physiol Biochem 2016;38:40-48. 


\section{Cellular Physiology Cell Physiol Biochem 2017;41:530-542

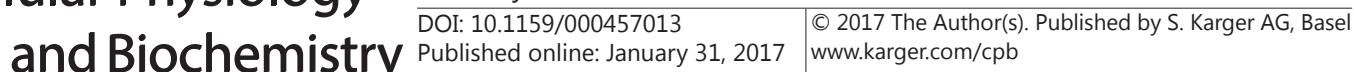

Wang et al.: MiR-125b Suppresses Osteogenic Differentiation of hBMSCs

25 Mizuno Y, Yagi K, Tokuzawa Y, Kanesaki-Yatsuka Y, Suda T, Katagiri T, Fukuda T, Maruyama M, Okuda A, Amemiya T, Kondoh Y, Tashiro H, Okazaki Y: Mir-125b inhibits osteoblastic differentiation by downregulation of cell proliferation. Biochem Biophys Res Commun 2008;368:267-272.

-26 Huang K, Fu J, Zhou W, Li W, Dong S, Yu S, Hu Z, Wang H, Xie Z: Microrna-125b regulates osteogenic differentiation of mesenchymal stem cells by targeting cbfbeta in vitro. Biochimie 2014;102:47-55.

27 Miyazono K, Maeda S, Imamura T: Bmp receptor signaling: Transcriptional targets, regulation of signals, and signaling cross-talk. Cytokine Growth Factor Rev 2005;16:251-263.

28 Derynck R, Zhang YE: Smad-dependent and smad-independent pathways in tgf-beta family signalling. Nature 2003;425:577-584.

29 Nohe A, Keating E, Knaus P, Petersen NO: Signal transduction of bone morphogenetic protein receptors. Cell Signal 2004;16:291-299.

30 Nakase T, Yoshikawa H: Potential roles of bone morphogenetic proteins (bmps) in skeletal repair and regeneration. J Bone Miner Metab 2006;24:425-433.

- 31 ten Dijke P, Yamashita H, Sampath TK, Reddi AH, Estevez M, Riddle DL, Ichijo H, Heldin CH, Miyazono K: Identification of type i receptors for osteogenic protein-1 and bone morphogenetic protein-4. J Biol Chem 1994;269:16985-16988.

- 32 Musgrave DS, Pruchnic R, Bosch P, Ziran BH, Whalen J, Huard J: Human skeletal muscle cells in ex vivo gene therapy to deliver bone morphogenetic protein-2. J Bone Joint Surg Br 2002;84:120-127.

-33 Wyatt AW, Osborne RJ, Stewart H, Ragge NK: Bone morphogenetic protein 7 (bmp7) mutations are associated with variable ocular, brain, ear, palate, and skeletal anomalies. Hum Mutat 2010;31:781-787. Wan DC, Shi YY, Nacamuli RP, Quarto N, Lyons KM, Longaker MT: Osteogenic differentiation of mouse adipose-derived adult stromal cells requires retinoic acid and bone morphogenetic protein receptor type ib signaling. Proc Natl Acad Sci USA 2006;103:12335-12340.

-35 Quarto N, Wan DC, Longaker MT: Molecular mechanisms of fgf-2 inhibitory activity in the osteogenic context of mouse adipose-derived stem cells (mascs). Bone 2008;42:1040-1052.

36 Xia HF, He TZ, Liu CM, Cui Y, Song PP, Jin XH, Ma X: Mir-125b expression affects the proliferation and apoptosis of human glioma cells by targeting bmf. Cell Physiol Biochem 2009;23:347-358.

-37 Kappelmann M, Kuphal S, Meister G, Vardimon L, Bosserhoff AK: Microrna mir-125b controls melanoma progression by direct regulation of c-jun protein expression. Oncogene 2013;32:2984-2991.

38 Urist MR: Bone: Formation by autoinduction. Science 1965;150:893-899.

-39 Guizzardi S, Di Silvestre M, Scandroglio R, Ruggeri A, Savini R: Implants of heterologous demineralized bone matrix for induction of posterior spinal fusion in rats. Spine (Phila Pa 1976) 1992;17:701-707.

40 Berven S, Tay BK, Kleinstueck FS, Bradford DS: Clinical applications of bone graft substitutes in spine surgery: Consideration of mineralized and demineralized preparations and growth factor supplementation. Eur Spine J 2001;10:S169-177.

41 Aghdasi B, Montgomery SR, Daubs MD, Wang JC: A review of demineralized bone matrices for spinal fusion: The evidence for efficacy. Surgeon 2013;11:39-48.

42 Einhorn TA, Lane JM, Burstein AH, Kopman CR, Vigorita VJ: The healing of segmental bone defects induced by demineralized bone matrix. A radiographic and biomechanical study. J Bone Joint Surg Am 1984;66:274279.

-43 Eryilmaz T, Ozmen S, Lortlar N, Omeroglu S, Ayhan S, Celebi C: Feasibility of demineralized bone matrix for craniomaxillofacial contour restoration. J Craniofac Surg 2011;22:1888-1892.

-44 Liu G, Li Y, Sun J, Zhou H, Zhang W, Cui L, Cao Y: In vitro and in vivo evaluation of osteogenesis of human umbilical cord blood-derived mesenchymal stem cells on partially demineralized bone matrix. Tissue Eng Part A 2010;16:971-982.

45 Kurkalli BG, Gurevitch O, Sosnik A, Cohn D, Slavin S: Repair of bone defect using bone marrow cells and demineralized bone matrix supplemented with polymeric materials. Curr Stem Cell Res Ther 2010;5:4956. 